\title{
Search Engine Optimization on PHP based web pages in practice
}

\author{
Snezana Savoska ${ }^{1}$, Niko Naka $^{1}$, Violeta Manevska $^{1}$, and Blagoj Ristevski ${ }^{1}$ \\ ${ }^{1}$ Faculty of Information and Communication Technologies \\ Partizanska bb 7000 Bitola, Macedonia \\ \{snezana.savoska, violeta.manevska, blagoj.ristevski\}@ fikt.edu.mk \\ naka_niko@yahoo.com
}

\begin{abstract}
SEO is a symbiosis of arts and science. The art's aspect of optimization requests dynamics, creativity and intuition in order to improve the searching rankings. The searching algorithms are too complex to make backward engineering to each aspect of them. Therefore, some scientific aspects have been included such as assumptions, notices, drawing conclusions and achieving multiplicative results as SEO functions. Ability to connect the users with the service or goods supplier is, probably the most significant and the most valuable skill placed on the Internet. In this paper we share our experience of using techniques and methods for improvements of SEO results through a practical example. Some important factors for rising of the rankings of web pages and SEO methodologies of "white and grey" hat, used to increase the ranking of the targeted web sites, have also been presented.
\end{abstract}

Keywords: SEO, White hat SEO, Grey hat SEO, PHP, on-page optimization, offpage optimization

\section{Introduction}

Searching for information on the Internet is not a simple activity, especially when we consider todays' up flood of information. Ranking of research results is an important business that leads the new Internet economy. For this reason, in this paper we will explain and apply some techniques and methods that are used to understand how indeed the "new" Internet search philosophy functions. It includes using of: social networking, blogging, optimizing, semantic rules etc.

The whole industry supported by professionals is in search for optimization and its use in research. This became especially obvious when the research engine with a funny name „Google“, was placed on the Internet [1]. This way, the entire philosophy of Internet changed and it became available to hundreds of thousands of clients and also created a customers' database of millions records of data [2]. At the same time, this was the fastest growing company in the history of business. In only a few years, Google has become a cultural phenomenon and an essential part of every intelligent, business-user strategy [3]. This and similar sites have developed the concept for the new technological intermediary economy of communication between those who search for products, services and information, and those who could provide them [1]. The abbreviation for 
this interaction has become a synonym for "finding", "need for learning", "experience and practice" and "use of research".

Searching should be understood as a different way of conversation with a good or potential client. The skills that each person naturally possesses, the approach to the service, environment in which they exist, all of them represent skills which need to be translated into the language of Search Engine Optimization (SEO) [3]. Although it may seem frustrating at first, in fact, it is a beginning of running business in some different way. For such strategy, one has to be ready and to know what has to be done to take the chance and avoid the risk of failure [4].

SEO could be described as a cluster of strategies and techniques which are used to increase the number of website visitors through gaining a high ranking placement on the website's search results [2]. The primary objective of SEO is to increase the number of website visitors and to convert those visitors into potential clients [4].

The paper emphasizes the various factors that should be taken into consideration while optimizing the website. Web pages that are used as examples for SEO are created with purpose of one of the authors' research for master thesis and were active from July 2013 to July 2015 (google history links). Various SEO techniques were used for increasing the page ranking of mentioned web sites, described in the paper.

The first section of the paper introduces and describes the needs of the research. The second part describes related works in this area and the third one describes SEO techniques. The next section explains SEO activities undertaken with purpose to increase web sites page ranking (PR). The fifth section summarizes the results of undertaken activities on the web pages and analyzes the empirical results of the mentioned activities. Conclusion and remarks make a summary of the paper.

\section{Related works}

Many efforts are made by researchers on SEO area. SEO is defined as strategies, methods and structures, as form of engineering and modifications of variables affecting the web site positioning in the results of search engines [4, 5]. In the beginning, companies offered their manuals for SEO and "marketing in digital era" $[2,3,4,7]$. Some of the researches were focused on SEO strategies $[8,5,9,10]$ and the others on SEO algorithms analysis [10-13]. Many of researches see SEO as a tool in the business world [2]. Considerable number of researches consider SEO trends and SEO algorithms development [10, 14]. Some of them consider SEO as art [1,3] and the others as technological secrets [6]. The fact is that SEO is becoming wide range industry, focused on the specific activities that provide better search engine positioning and bring many advantages for the users that use SEO.

According to some authors $[9,14,15]$, the main classification of SEO techniques can be seen as On Page SEO and Off Page SEO. Focusing of the search engine, they can be classified as Black Hat SEO, White Hat SEO and Grey Hat SEO [9, 16].

On Page SEO and Off Page SEO promote some techniques and methods to increase the level of page ranking of the web site. Considered papers state that, around $70 \%$ of the job can be done with On Site SEO [9] which includes optimization of HTML code, web site content, architecture, text optimization, tags, URL structure, hyperlinks, headers, picture alternate text or tags and etc. The other $30 \%$ of all SEO processes are 
dedicated to the activities linked with inbound links, connected with the contents on the web sites. These activities include link building, article writing, blog publishing, social media bookmarking, web site activity as directory submission sites, forum posting etc. [9]

Black, White and Grey Hat SEO are consequently illicit, recommended and dubious techniques if they are seen from the search engine (SE) aspect. Black Hat SEO is focused on usage of techniques for quick high return business model and can be sanctioned by SE sandboxing. Authors mention techniques as keywords stuffing, doorway pages hidden text, adding unrelated keywords break the SE rules and are irregular for usage $[16,14,9,21]$.

According to some authors, White Hat SEO are some kind of SEO guides and policy $[14,17,5,1]$. White Hat SEO include using keywords and keyword analysis, meta tags, unique content, back linking, site maps, robots, link building to increase the visibility of the web site and search engine result page (SERP) [9].

Researchers mention Grey Hat SEO techniques as something between White Hat SEO and Black Hat SEO [5]. Grey Hat SEO include actions such as cloaking, usage of old domains, duplicate content, purchasing links, guest posting, social media automation and buying followers. Cloaking includes altering meta-information and doorway pages that manipulate bots and trick users, as the others mentioned techniques that are in accordance with SE rules, but sometimes can be sanctioned by the SE [5].

Past SEO experience is connected with week unethical search algorithms contrary to present SEO that is connected more with algorithms that are intelligent and use state of the art techniques by SEO specialists $[10,18,20]$. This means, web sites have to be semantic, with optimized keywords and structure, unique and optimized content and optimized links. Now, SEO measures and ranks quality of content from relevant to irrelevant, natural back links, site popularity and activity of social media, freshness of the content, sometime voice and mobile search, capability for personalized search, title, headings and image tags. Then, using methods of web structure mining, web content mining and web usage mining and data analysis [14], which help calculate the parameters that create page ranking (PR).

There are many papers and books trying to explain SE algorithms that are very complex and require huge hardware performance as well as database servers and fast memory storages [6, 15]. Apart from hardware performance and excellent SE algorithms, it is important to have a skillful person who has excellent knowledge of SEO techniques.

\section{Review of SEO techniques}

SEO includes inside and outside website analysis, involving creation of links, corresponding architecture and website development, unique content, competitive analysis, key words research, content development and many other tasks [1]. Although the website content is essential to its popularity, Google's ability to count the incoming links is considered to be a huge advantage in optimization. SEO provides numerous conveniences: the best results from website search on the Search Engine Result Page, historical trust factor, lower ownership costs and other conveniences. 
According to SEOmoz and research leading SEO factors, the most important ranking factors (http://www.seomoz.org/article/search-ranking-factors) are: using keywords in the title tag, anchor text of incoming links, global authoritative links on the website, website age, the popularity of link within the internal structure of the website, thematically relevant incoming links, popularity of links on the website in the thematic community, using key words in the text body and popularity of global links on websites that link to your website.

There are mainly three SE optimization techniques that can be used for improving PR. Black hat SEO is the practice that increases the ranking of your website by means that violate the rules of the search engines and are considered unethical. Many Black hat SEO tactics are applied although penalized by search engines if discovered. They are: automated content, doorway pages, hidden text or links, reporting of competition, concealment, sneaky redirects and others. Another famous Black hat SEO techniques are: manipulation of links - buying links, schemes with links, turning the articles, link farms, network links, link wheels, rich selected spam, automatically searches on Google, creating pages, sub domains, or domains with dual content, parties with malicious behaviors such as fishing, viruses, Trojans, and other mal-ware [6, 3].

Optimization techniques White hat SEO include supplying customers with quality and accurate content that is relatively well organized. Using relevant keywords in the title tag, $\mathrm{H} 1$ tag and anchor text of incoming and out coming links are examples of white hat SEO [16]. In the SEO activity we used White Hat SEO and Grey Hat SEO.

Gray hat SEO is an optimization technique that differs more from White hat SEO and is a questionable set of optimization techniques like double posting content in different places, irrelevant links providing three-way linking, reciprocal linking and using paid links and viral marketing [14]. Some of the activities made for rising PR can be classified as Grey Hat SEO.

All of them have the same objective - improvement of visibility of the website on the Internet, but differ in the way they lead to that objective. The fact is that the relevance of each factor mentioned before increases and it is important for today's SEO.

\section{SEO activities and prerequisites}

First off all, we planned SEO activity. Creation of web sites in Macedonian and in English language is the first activity. Websites (food-flavour.com and farmer.com) ${ }^{1}$ intended for the project are created with usage of PHP and MySQL-based CMS (Content Management System), with WordPress that is safe and SEO-friendly tool. Web sites farmeri.mk was active from July 2013 to July 2015 and food-flavour.com from May 2014 to May 2015. In the beginning the web sites PR was zero (PR0).

The purpose of the project was to raise the websites page ranking, which means to ensure it appears higher in the search engine results for a given set of keywords. To achieve this, designers have to understand CMS systems and how it can be configured to get a better page ranking. Also, designers should be familiar with the influence of

\footnotetext{
${ }^{1}$ The web sites' history can be seen at links of web archive: https://web.archive.org/web/20140604082755/http://www.food-flavors.com/ and https://web.archive.org/web/20130819021542/http://www.farmeri.mk/
} 
social networking, using of sitemap, tags, metatags and related links for raising the PR. Website optimization on PHP web pages involves editing of its content as well as HTML and PHP coding to increase the relevance for specific keywords and remove barriers for indexing by search engines algorithms. The promotion of the website with more background or incoming links is another SEO tactic that contributes to raising PR.

Getting the higher position, i.e. in the first page of the search engines results for selected keywords, is a great result for a website designer, because there are millions of web pages that compete and are ranked by web search engines. For purpose of raising mentioned websites ranking, we used some of the SEO techniques that were taken in consideration previously. For all these assumptions, we plan the next activities in phases, leading from the mentioned needs and White Hat SEO recommendations.

For aforementioned research, dependent variables will be websites' traffic and page ranking. The plan is to use the following as independent variables: websites' number of visitors, number of nested background links, number of complex keywords, number of visits coming from background links, number of visits by organic results of complex keywords etc.

\subsection{White Hat SEO assumption and activities to prove}

We start from the assumption that PR of websites will be improved by placing outgoing links and using complex keywords to quickly find the page. The expected result will be increased traffic to the website. The more visitors the web page has, the higher is its ranking and the interest in placing ads which will bring profits through advertising.

Background links. To prove these assumptions, two background links were placed on the websites that have .edu domain: famis.edu. $\mathrm{mk}^{2}$ and fbn.uklo.edu.mk. Both websites possess PR4, so for a period of 6 months, when it was time for a new update of website ranking, farmeri.mk reached PR3. This result is presented in Figure 1b. This was preceded by the period from website creation (10/07/2013) until 12/10/2013, when the website had a PR 0 or N / A (Fig. 1a).

By placing the background links that point to the homepage of farmeri.mk and keyword "tips for Agriculture" and in Macedonian language "soveti za zemjodelstvo", the number of visits increased. Table 1 shows a comparison of the daily visits with and without background links. The number of visitors was calculated by using Google Analytics, so by posing background connections and other optimization techniques, the number of visitors was- 12,234 in one year, so we can confirm the preset assumption.

Usage of fun pages on social networks. The next assumption is that ranking of the website was greatly affected by creating fan pages on social networks by increasing the number of visitors through regular publications of posts. With increased number of members in the fan pages, usage of links to related articles are also increased. The

2 Website of Faculty for administration and information systems management, St. Kliment Ohridski University (FAMIS) existed until 21.10.2014 and can be seen from website history site at link: https://web.archive.org/web/20130905072921/http://www.famis.edu.mk/w3/ 
website traffic with usage of this technique is also increased. A fan page on Facebook social network was created on 05.8.2013, and by constantly posting new content, available to those who "like" the fan page, the number of visitors increased by 139 "likes" - Fig.2.

Table 1 Comparison of average number of daily visits, with and without posted background links

\begin{tabular}{|c|c|}
\hline $\begin{array}{c}\text { farmeri.mk without background links } \\
\text { (average number of daily visitors in the } \\
\text { period 15.07.2013-15.09.2013) }\end{array}$ & $\begin{array}{c}\text { farmeri.mk with background links } \\
\text { (average number of daily visitors in } \\
\text { the period 20.09.2013-29.12.2013) }\end{array}$ \\
\hline 30 & 120 \\
\hline
\end{tabular}
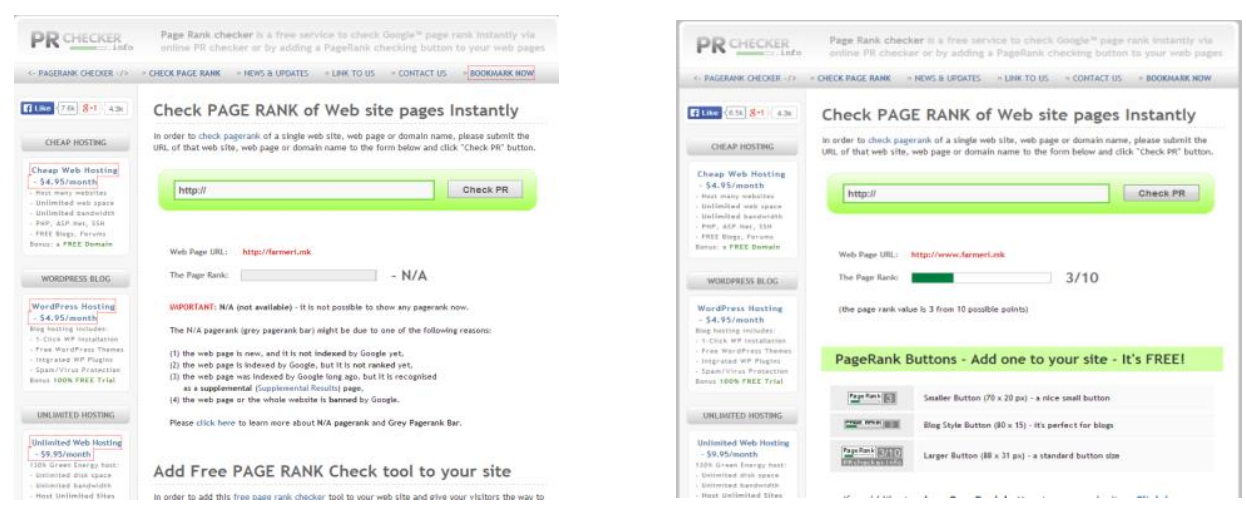

Fig. 1a) PR 0 (N/A) from the date of creation of the website 1b) Result obtained on December 2013

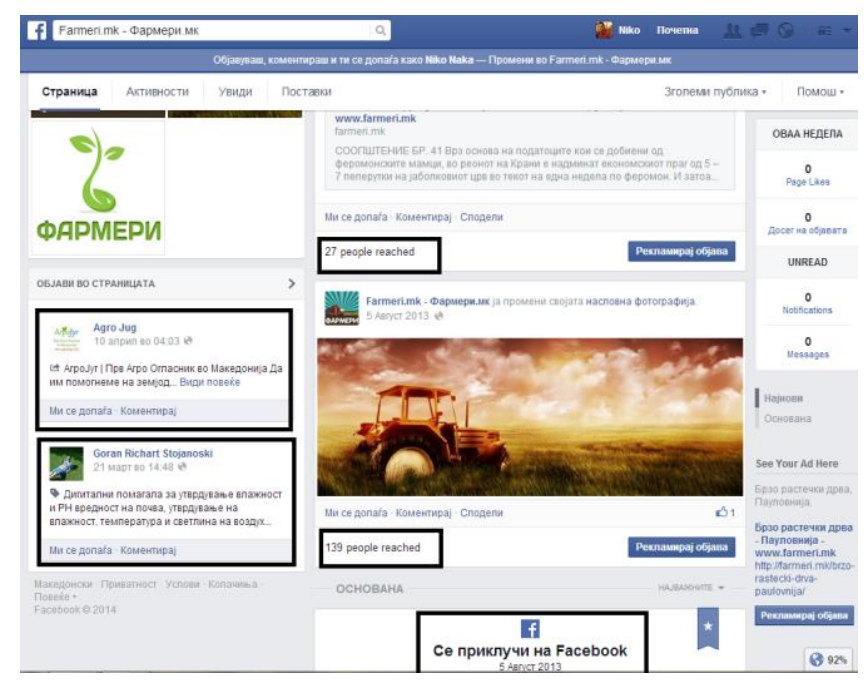

Fig. 2 Fun pages of farmeri.mk on the Facebook social network 
Creation of XML Sitemap and robot.txt. The next theses will be: creation of XML Sitemap and robots.txt file also influence page ranking because they allow "spiders" to index page by search engines like Google and Bing in which $97 \%$ of searches take place. By applying these techniques, obtained results confirmed our thesis. Table 2 shows the comparison before and after using the Sitemap of the English version of the website - food-flavors.com

Table 2 Comparison before and after using of Sitemap of webpage food-flavors.com

\begin{tabular}{|c|c|}
\hline $\begin{array}{c}\text { Before Sitemap of food-flavors.com } \\
\text { creation (number of indexed links) }\end{array}$ & $\begin{array}{c}\text { After Sitemap of food-flavors.com } \\
\text { creation (number of indexed links) }\end{array}$ \\
\hline 3 & 102 \\
\hline
\end{tabular}

Selection of keywords. Keywords' analysis was made with keyword planner from Google AdWords. For this reasons Google AdWords account has to be created. Then, in the section "Search for keyword and add group idea", complex keywords have to be entered and then, we can get some ideas for targeting websites and some other specifications. The price list for specific keywords is available on the site and they are related to AdWords.

Once you choose keywords, the next step is setting original content, updated all the time with new information. Checking originality of the contents was made by choosing http://www.duplichecker.com/. URL or text up to 1500 words need to be entered and then select "search". If there is some plagiarism found, the source will be displayed, otherwise there is message that informs the content is unique.

Installation of plug-ins. The next assumption is that there are many free plug-ins that can be installed to help website optimization. Using WordPress as a CMS for creating of mentioned web pages, two different plug-ins for SEO were installed: All in One SEO for website food-flavors.com, and YOAST for farmeri.mk. Generally, both plug-ins have same attributes with difference that YOAST can create a Sitemap and connection with Google Analytics [17].

Applying for placing ads on AdWords. Applying for placing ads on AdWords is very important for page ranking, states the next theses. Because Macedonian language is not yet in the list of possible languages, we applied only for English version of website, food-flavour.com. Our first request for placing ads was refused by Google with explanations (Fig. 3).

After some improvements made on food-flavoour.com, according to service requirements and criteria listed in received mail as well as after analysis of other websites collaborators of AdSense, our application was accepted. The application is associated with data monitoring of advertising screen with four tabs: Home (account balance), My ads (created ad units and dimensions of ads), Blocking ads (cheaper click value) and Performance reports that show reports about the visitors' destinations and day's ads average rate. An important element is the Page click through rate that can be calculated with the following formula: 


$$
C T R=\frac{C P C}{C P M} * 100
$$

wherein $C T R=$ click through rate, $C P C=$ cost per click, and $C P M=$ cost per mile. Better indexing with Sitemap was created with All in One SEO plugin (Fig. 4).

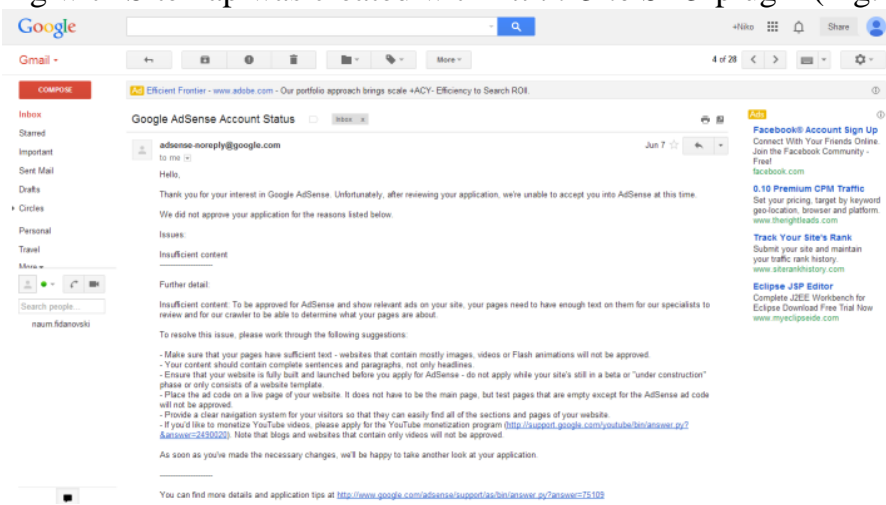

Fig. 3 E-mail message with rejected demand for placing ads

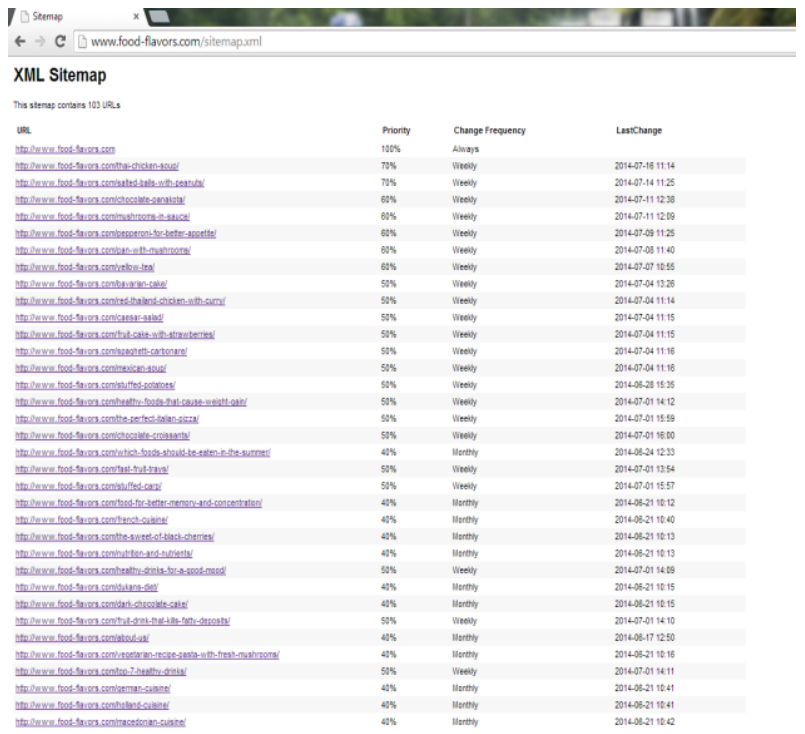

Fig. 4 Sitemap of website food-flavors.com

Influence of page loading time to site optimization. Page loading time also affects site optimization. Websites that get faster are ranked higher in the search engines' results. The loading speed can be calculated with internet service http://tools.pingdom.com/fpt/ by simply typing in the URL. Fig.5 shows results for 
website food-flavors.com, submitted on the mentioned link that shows that loading page is faster than $46 \%$ of tested websites.

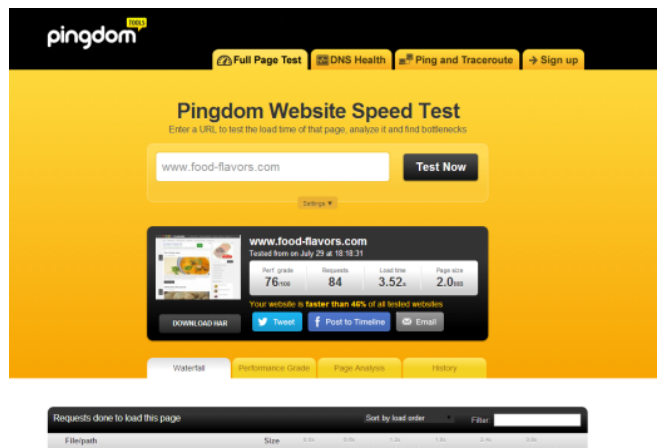

Fig. 5 Website pingdom for testing of loading speed

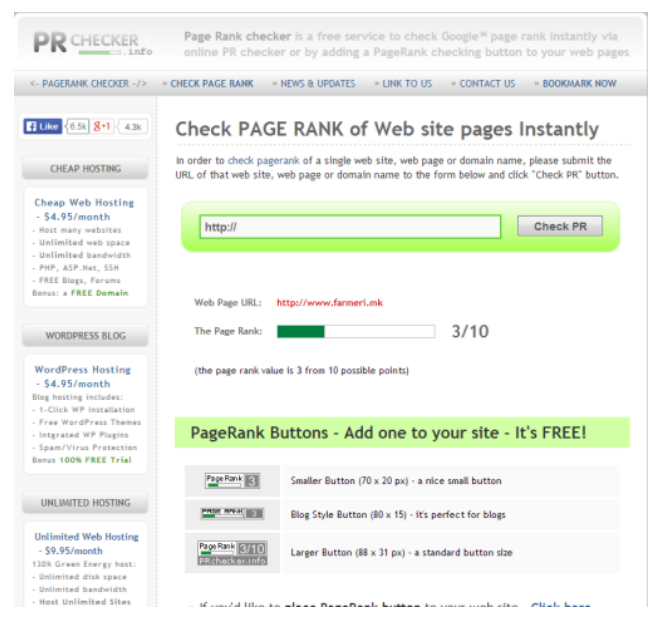

Fig. 6 Check of page ranking (PR) of website with add-ins

Other competition factors . Macedonian version of mentioned sites, farmeri.mk, was created a year earlier than English version. Logically, it is easier to find it in the top five results for a particular keyword. A number of factors, such as less competition, lower number of websites that compete for that keyword, influence faster PR raising. Links to some Macedonian websites, especially those containing gov extension can lead to authoritarian websites. Checking the page ranking of the website can be made through services such as http://checkpagerank.net/ or through the installation of add-ins for web search engines (Fig.6). This means lower competition of complex keywords will increase PR. By using keywords in Macedonian language „совети за земјоделство“, the Macedonian version of the website managed to appear as a first result in the search for these keywords on Google search engine. 


\subsection{Grey Hat SEO activities}

In obtaining the objectives of the project to achieve a better page ranking and reaching higher positions in search engine results, besides standard optimization techniques including the White hat SEO, some of the Gray hat SEO techniques were used. This included a campaign on the website microworkers.com in which those who will carry out anticipated tasks will be adequately rewarded. The request was to visit FAMIS ${ }^{3}$ website, to find the site banner of farmer.mk and spend at least 1 minute on the site. All activities described in those requirements are shown in Fig. 7.

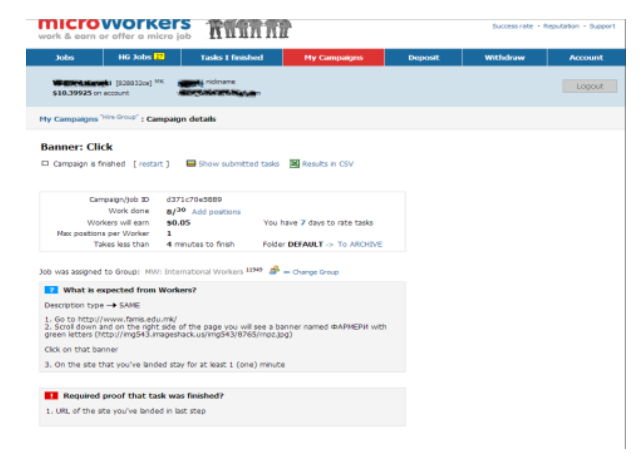

Fig. 7 Created campaign on website microworkers.com

As an advertising place for the website's promoting was the Facebook fan page, through which the latest posts placed on the site were published. With this activity the number of visits increased, by those who "like" the page (Fig. 8).

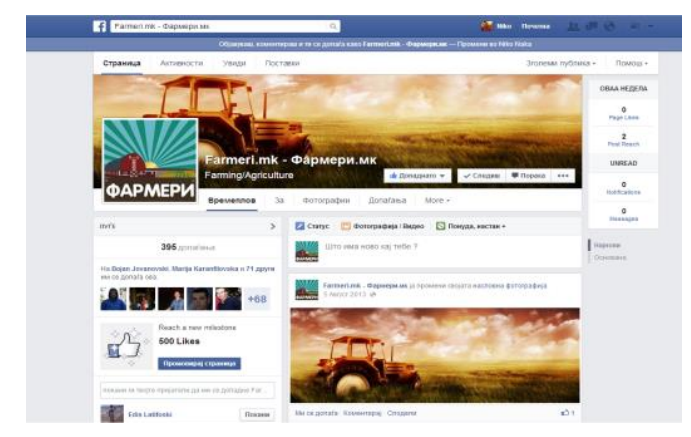

Fig. 8 Facebook fun pages for farmeri.mk

Indexing of websites by web search engines have to be allowed through the file robots.txt. Once you make the questionnaire for search links on webpage and how many links are indexed, you get the result that only one link is found. If we have to index

3 FAMIS webpage history can be seen at link: https://web.archive.org/web/20130905072921/http://www.famis.edu.mk/w3/ 
webpage, we have to use code shown in Fig. 9. The code shows that the indexing is not allowed only for folders wp-admin and wp-includes, but the others can be indexed.

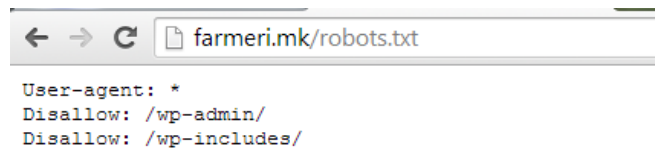

Fig. 9 Example for robots.txt - file for website indexing

\section{Google Analytics of visits of website}

To have a complete review of the visits of the website, a time period that the users spent on the website, users' destination and the other statistics, we have to relate our websites with Google Analytics, a service that provides tracking and website analytics. Linking with Google Analytic and analysis obtained from service are shown in Fig. 10. There is data as number of sessions that have been made for website farmeri.mk, number of users, reviews and average user's retention on the website. For website farmeri.mk, the Sitemap was created with Yoast plug-in (Fig.11).

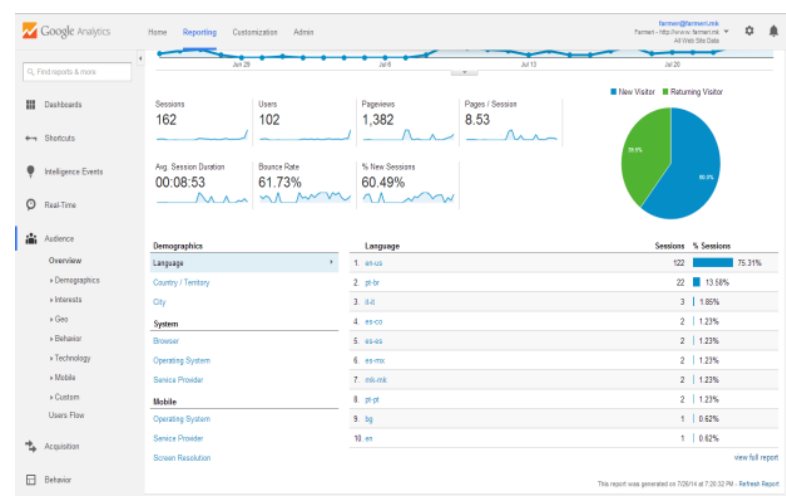

Fig. 10 Google Analytics analysis of website statistics - demography

\section{Conclusions}

SEO are methods and techniques for improvement of website' page ranking and increasing its visibility. Search algorithms are too complex to make reverse engineering, so SEO have to be partly science, partly art. SEO include, among inventive design, usage of scientific aspects as assumptions or thesis testing, observations, drawing conclusions and reproducible results [19]. These two aspects of SEO are guidelines for 
issues on how SEO will develop in the future. It is not enough for SEO to make adjustment for change, because they need to cause the change. Successful SEO professionals have to play the role of agents of changes.

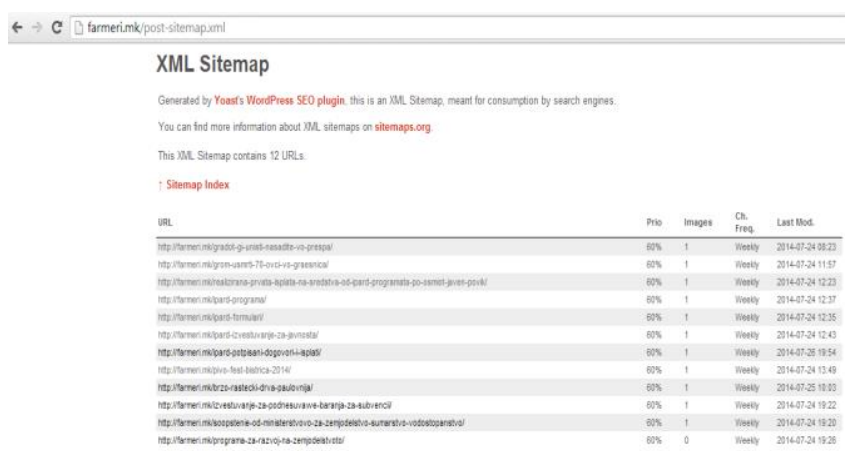

Fig. 11 Sitemap for website farmeri.mk, created with YOAST plug-in

With some of the White hat SEO and Gray hat SEO techniques we improved page ranking of newly created websites and for the mentioned period of time, we confirmed the assumptions set. To increase web traffic and consequently increase PR, we did activities from White Hat SEO and Grey Hat SEO.

From White Hat SEO activities, we placed background links to authoritative websites and created fun pages on social networks. Also we created XML sitemap and robots.txt file, made complex keywords, created AdWords account, posted original contents. Other activities included: installing free plug-ins, applying for posting ads on AdWords, calculating click through rate on the pages and optimizing time for page loading.

From the box of Grey Hat SEO activities, we made a campaign on the other websites with some task that includes a visit to our websites, created advertising webpage on Facebook and allowed indexing of almost all contents of websites in robot.txt. Finally, we related websites with Google analytics and gained Google analytics and websites analysis. With these activities we increased the PR from PR0 to PR3.

Needs of companies or any organization to find new customers would not decrease, a contrary, the need for searching, quick access to information, products and services will increase all the time. These trends will require increased usage of SEO. Certain rapid technological development may require another kind of SEO or it will be replaced by another type of technology, perhaps Watson, cognitive technology that processes information in a way that a human brain resonates [8] and which understands natural languages and generates hypotheses based on evidence and learning. According to initial clarifications, Watson is "smart" in three ways: by learning from its customers, from previous interactions and by generating new information. Organizations will be able to fully understand and use the information they are surrounded by and use data to improve decisions.

It is thought that Watson will include several areas, and thus the biggest search engines will begin to use it, changing search engines' algorithms daily and fighting against Black hat SEO $[9,21]$. Watson will be smart enough to decide for websites that have to be on the first page of the search engine's results without the need to use different techniques. For these reasons, our SEO activities, in the future, will be 
connected to studying these new SEO algorithms and finding ways to influence the increase in PR with some practical activities on the targeted websites.

\section{References}

1. Elmansy, R.:Teach Yourself Visually Search Engine Optimization. ISBN: 978-1-118-470664, Wiley. (2013)

2. The Google AdSense Handbook, [Online] https://www.free-ebooks.net/ebook/Build-AnAdSense-Empire/pdf?dl\&preview. (Available Jan 2016)

3. Enge, E., Spencer, S., Fishkin, R., Stricchiola J.C.: The art of SEO. ISBN:978-0-596-518868, O'Reilly. (2009)

4. The Google AdSense Empire Handbook, Free Google Book, [Online], http://www.maxblogpress.com/oto/dn15sw2jk5/ais/AdsenseEmpire.pdf. (Available Jan 2016)

5. Saravanakumar, S., Ramnath, K., Ranjitha, R. Gokul, V.G.: A New Methodology for Search Engine Optimization without getting Sandboxed. International Journal of Advanced Research in Computer and Communication Engineering, Vol. 1, Issue 7, ISSN : $2278-1021$. (2012)

6. Adams, R.L.: SEO Black Book: A Guide to the Search Engine Optimization Industry's Secrets. ISBN: 978-1-482-66516-1, Copyright @ Adams. (2013)

7. Stones, R.: eMarketing, The Essential guide to Marketing in a Digital World. 5-th edition, Quirk, ISBN: 978-0-620-56515-8. (2013)

8. Beel, J., Gipp, B., Wilde, E.: Academic Search Engine Optimization (ASEO): Optimizing Scholarly Literature for Google Scholar \& Co. Journal of Scholarly Publishing University of Toronto Press, Vol. 41, No. 2, 176-190. (2010) doi: 10.3138/jsp.41.2.176

9. Poongkode, J.P.S., Nirosha, V.: A Study on Various Search Engine Optimization Techniques. International Journal of Innovative Research in Computer and Communication Engineering, Vol. 2, No. 11. (2014)

10. Saberi, S., Saberi, G., Mohd, M.: Past, Present and Future of Search Engine Optimization, ISBN: 978-0-9891305-2-3, [Online] www.sdiwc.net/digital-library/web-admin/uploadpdf/00000797.pdf, (Available 21.1.2016)

11. Wang, D.Y., Savage, S., Voelker, J.M.: Juice: A Longitudinal Study of an SEO Botnet, University of California, San Diego [Online] http://cseweb.ucsd.edu/ dywang/pubs/juicendss13.pdf, (Available Jan 2016)

12. Bedi, G.S., Singh, A.: Analysis of Search Engine Optimization (SEO) Techniques, International Journal of Advanced Research in Computer Science and Software Engineering, Vol. 4, No. 3. (2014)

13. SEOmoz Inc, [Online] http://moz.com/google-algorithm-change (Available 15.03.2014)

14. Singh, E.T., Maini, R.: A Comprehensive Review on Search Engine Optimization, Journal of Global Research in Computer Science, Vol. 4, No. 1. (2013)

15. Wang, F., Li, Y., Zhang, Y.: An empirical study on the search engine optimization technique and its outcomes. In proceedings of 2nd International Conference on Artificial Intelligence, Management Science and Electronic Commerce (AIMSEC), 2767-2770. (2011) [Online] http://ieeexplore.ieee.org/xpl/login.jsp?tp=\&arnumber=6011361\&url=http\%3A\%2F\%2Fieee xplore.ieee.org\%2Fxpls\%2Fabs_all.jsp\%3Farnumber\%3D6011361, (Available Jan 2016)

16. Black Hat and White Hat SEO, Confluence, Seattle. (2011) [Online] http://www.confluencedigital.com/wp-content/uploads/2011/03/Black-Hat-White-HatSEO.pdf, (Available 25.1.2016)

17. Amerland, D., Google ${ }^{\mathrm{TM}}$ Semantic Search, by Pearson Education, Inc, ISBN-13: 978-0-78975134-8. (2014)

18. Mandhu, G., Gorardhan, D.A., Rajinikanth, T.V.: Intelligent Semantic Web Search Engine: A Brief Survey. International Journal of Web \& Semantic Technology, Vol.2, No.1. (2011) 
19. Shih, B.Y., Chen, C.Y., Chen, Z.S.: An Empirical Study of an Internet Marketing Strategy for Search Engine Optimization. Human Factors and Ergonomics in Manufacturing \& Service Industries, Vol. 23, No. 6, 528-540. (2013)

20. How Stuff Works Tech. [Online] http://computer.howstuffworks.com/semantic-web.htm (Available 11.04.2015)

21. Howard, F., Komili, O.: Poisoned search results: How hackers have automated search engine poisoning attacks to distribute malware. SophosLabs technical paper. (2010) 